\title{
UAV-BASED STRUCTURAL DAMAGE MAPPING - RESULTS FROM 6 YEARS OF RESEARCH IN TWO EUROPEAN PROJECTS
}

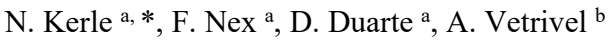 \\ ${ }^{\text {a }}$ Faculty of Geo-Information Science and Earth Observation (ITC), University of Twente, 7500 AE Enschede, The Netherlands - \\ (n.kerle, f.nex, d.duarte)@utwente.nl \\ b Experian, Singapore 339510 - anand.vetrivel@experian.com
}

KEY WORDS: Drone, computer vision, point clouds, machine learning, CNN, first responder, RECONASS, INACHUS

\begin{abstract}
:
Structural disaster damage detection and characterisation is one of the oldest remote sensing challenges, and the utility of virtually every type of active and passive sensor deployed on various air- and spaceborne platforms has been assessed. The proliferation and growing sophistication of UAV in recent years has opened up many new opportunities for damage mapping, due to the high spatial resolution, the resulting stereo images and derivatives, and the flexibility of the platform. We have addressed the problem in the context of two European research projects, RECONASS and INACHUS. In this paper we synthesize and evaluate the progress of 6 years of research focused on advanced image analysis that was driven by progress in computer vision, photogrammetry and machine learning, but also by constraints imposed by the needs of first responder and other civil protection end users. The projects focused on damage to individual buildings caused by seismic activity but also explosions, and our work centred on the processing of 3D point cloud information acquired from stereo imagery. Initially focusing on the development of both supervised and unsupervised damage detection methods built on advanced texture features and basic classifiers such as Support Vector Machine and Random Forest, the work moved on to the use of deep learning. In particular the coupling of image-derived features and 3D point cloud information in a Convolutional Neural Network $(\mathrm{CNN})$ proved successful in detecting also subtle damage features. In addition to the detection of standard rubble and debris, CNN-based methods were developed to detect typical façade damage indicators, such as cracks and spalling, including with a focus on multi-temporal and multi-scale feature fusion. We further developed a processing pipeline and mobile app to facilitate near-real time damage mapping. The solutions were tested in a number of pilot experiments and evaluated by a variety of stakeholders.
\end{abstract}

\section{INTRODUCTION}

\subsection{Structural damage mapping with remote sensing}

The first documented systematic post-disaster damage assessment attempt with remote sensing technology dates back to 1906, when earthquake-affected San Francisco was mapped with a $20 \mathrm{~kg}$ kite-borne camera (O'Rourke et al. 2006). This makes it one of the oldest applications in this domain, but also one of the few that continues to elude robust operational solutions. Since the early pioneering days nearly every type of active and passive sensor has been mounted on airborne platforms that range from tethered to autonomous or piloted, as well as satellites operating in different orbital or network constellations, to attempt increasingly automated damage detection (Dong and Shan 2013, Kerle 2015). However, despite more than a century of research and tremendous technological developments, operational image-based damage mapping, such as through the International Charter "Space and Major Disasters" or the Copernicus Emergency Management Service (EMS), continues to be a largely manual exercise (e.g., Belabid et al. 2019, Novikov et al. 2018).

Charter and EMS activations center on a particularly challenging type of damage mapping. The first maps are expected to be available within hours after image acquisition, while the particular damage pattern and its recognition is subject to a number of variables. Building types, configurations and construction materials differ, and recognizable damage indicators are strongly dependent on hazard type. Image type, in terms of spectral characteristics, incident angle and environmental conditions such as haze or cloud cover, further challenge the development of generic and widely applicable damage detection algorithms. Satellite-based damage mapping has the additional disadvantage that damage that may be quite variably expressed on each of the building's facades, its roof, as well as its interior, is largely reduced to a single dimension, the roof. Damage detection in reality is then supported by use of proxies, such as evidence of nearby debris or damage clues associated with particular shadow signatures (Kerle and Hoffman 2013). There have been some notable successes in satellite-based damage mapping, in particular related to cases where radar data have an advantage, in particular interferometric ( $\mathrm{Lu}$ et al. 2018) and polarimetric synthetic aperture radar ( $\mathrm{Li}$ et al. 2018). Methods to process optical data using advanced machine learning algorithms, including deep learning, have recently started to emerge (e.g., Sublime and Kalinicheva 2019), though still tend to focus on disaster-related changes rather than specific building-level damage mapping. While automated satellite-based damage mapping has shown limited progress, the proliferation and rapidly growing maturity of unmanned aerial vehicles (UAV/drones) has created vast new prospects for rapid and detailed damage assessment. The work reported on here directly built on earlier experiences with the airborne Pictometry system that yields similar oblique, overlapping and multi-perspective imagery, while being less flexible and economical. Those images have been photogrammetrically processed (Grenzdorffer et al. 2008), and also used for structural damage assessment (Gerke and Kerle $2011 \mathrm{a}, \mathrm{b})$. In 2013 we began working on two European-funded projects that focused on detailed damage mapping with UAV, and which are introduced below. 


\subsection{The RECONASS and INACHUS projects}

RECONASS (Reconstruction and Recovery Planning: Rapid and Continuously Updated Construction Damage, and Related Needs Assessment; www.reconass.eu) and INACHUS (Technological and Methodological Solutions for Integrated Wide Area Situation Awareness and Survivor Localisation to Support Search and Rescue Teams; www.inachus.eu) are research projects funded through the $7^{\text {th }}$ Framework of the European Union, and which ran with some overlap from 2013 until the end of 2018. The focus of RECONASS was to create a system for monitoring and damage assessment for individual high-value buildings, based on a range of internally installed sensors that included accelerometers, inclinometers and position tags, with data getting processed in a finite element structural stability model to determine damages caused by seismic activity or by either interior or exterior explosions. The contribution of our group focused on UAV-based 3D reconstruction of the building exterior and detailed damage mapping, to patch data gaps caused by failed sensor nodes, as well as to validate model outputs. The progressively developed methods were tested in a series of experiments, culminating in a pilot where a 3-story reinforced concrete building was first subjected to an explosion of $400 \mathrm{~kg}$ TNT placed about $10 \mathrm{~m}$ away, and later by a $12 \mathrm{~kg}$ charge detonated within the structure itself. End users, including the German Technisches Hilfswerk (THW), were present to assess the utility of the system.

The purpose of INACHUS was to assist disaster response and urban search \& rescue forces by providing early and increasingly detailed information on damage hotspots and the likely location of survivors. Broadly, this was achieved by a methodology that commenced with a wide-area assessment and scenario-based synthetic damage modelling, and then focused on identified hotspots with a range of instruments, including different UAV, ground-based and portable radar instruments to detect trapped survivors, as well as a robot to penetrate a damaged structure. Our focus was on scene reconstruction and damage mapping based on optical imagery from a low-cost UAV. Another partner, the French remote sensing lab ONERA, also deployed various larger UAV that carried different laser sensors, in part with proprietary solutions. The major pilots were assessed by a group of end users. More information about the projects can be found on the websites listed above.

\section{UAV-BASED DAMAGE MAPPING}

\subsection{Texture and segmentation-based approaches}

The earliest attempts to use UAV data in RECONASS were based on segmentation- and texture-based analysis of monotemporal imagery. Fernandez Galarreta et al. (2015) processed multi-perspective UAV imagery of an 2012 Emilia Romagna (Italy) earthquake site into detailed 3D models. Those were analysed for geometric damage indicators such as slanted walls or deformed roofs, as well as presence of debris piles (Figure 1). In addition object-based image analysis (OBIA) was carried out on the images to extract damage features such as cracks or holes, but also identification of those damages intersecting with apparent load-carrying structural elements. In recognition of the diversity and ambiguity of the observed damage patterns the work did not aim at automatic damage classification, except in cases where the 3D model clearly showed complete collapse (D5 on the European Macroseismic Scale 1998, EMS-98; Grünthal 1998). For all other cases the geometric and imagederived information was combined to produce a detailed damage score per façade, and in turn per building.
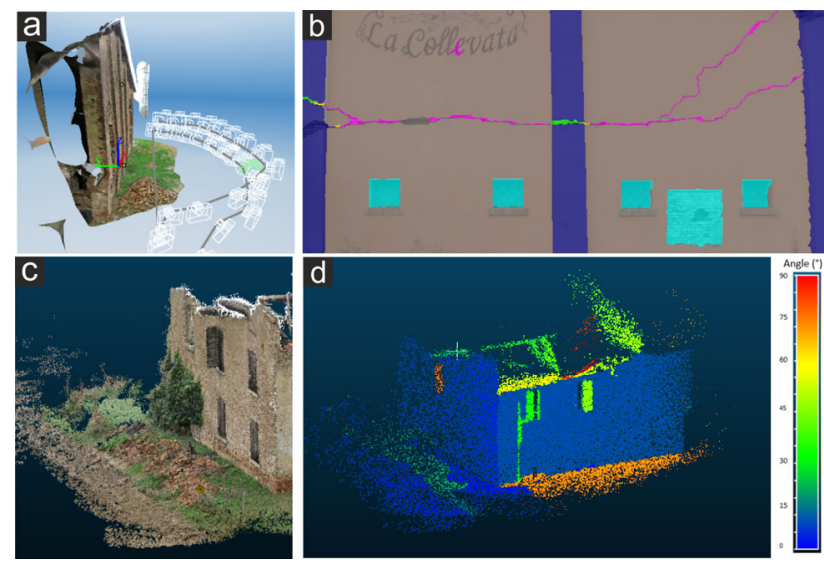

Figure 1. Damages identified from UAV-derived point clouds and from OBIA processing. (a) inclination in walls, (b) openings (turquois), cracks (magenta) and damage crossing beams, (c) and (d) detailed point cloud and segment orientation angles (adapted from Fernandez Galarreta et al. 2015)

The work of Fernandez Galarreta et al. demonstrated the significance of geometric information, in particular of openings. Vetrivel et al. (2015a) advanced the work by developing a method to isolate individual buildings from a detailed imagederived point cloud covering a neighbourhood of Mirabello (Italy) comprising nearly 100 buildings. Each of those was then subjected to a search for openings attributable to seismic damage, such as partial roof collapses or holes in the façades. The gaps were identified based on the Gabor wavelets as well as a histogram of gradient (HoG) orientation features. Two basic machine learning algorithms, Support Vector Machine (SVM) and Random Forest, were used to identify damaged regions based on the radiometric descriptors, with a success rate of approximately $95 \%$. However, in particular the segmentation of point clouds is frequently hindered by artefacts and data gaps. Therefore, we also developed an approach to project initial point cloud-derived 3D segments into image space, with a segmentation using both geometric and radiometric features yielding more accurate and complete building segments (Vetrivel et al. 2015b). Given that video-data are often the first acquired dataset of a disaster scene, be it by the police or news media, we also showed how also lower-quality video data can be analysed for damage with computer vision and segmentation methods (Cusicanqui et al. 2018).

Both early studies described above only used post-disaster imagery. However, even during the few years of our research projects the availability of high resolution pre-event reference imagery grew rapidly. This led to additional methodological developments that built on the segmentation- and texture-based damage detection described above, extending them into a multitemporal framework. In Vetrivel et al. (2016a) we used pre- and post-earthquake data of L'Aquila (Italy), and focused on the identification of $3 \mathrm{D}$ segments missing in the post-disaster data as an indicator of damage. We tested both voxel- and segmentbased approaches, finally settling on a composite segmentation method that subjects an integrated pre- and post-event point cloud to plane-based segmentation. While the work successfully advanced the damage identification to a robust change detection framework, the damage mapping itself remained a binary assessment. This mirrors a broader development. Early efforts at damage assessment frequently used satellite imagery to identify damaged areas more generally, while airborne data were used to detect specific damage features, usually debris piles (e.g., Mitomi et al. 2000). Later works increasingly attempted to map different damage levels on the EMS-98 scale 
(e.g., Corbane et al. 2011, Dubois and Lepage 2014, Yamazaki et al. 2005). However, it became increasingly clear that especially lower damage levels, negligible, moderate and substantial (D1-D3, respectively), tend to be very hard to identify in imagery, hence lower grades were aggregated, continuously moving towards the binary classification system of damaged vs. undamaged again.

\subsection{Advanced classifiers and hand-crafted features}

Vetrivel et al. (2015a) had shown the utility of HoG and Gabor filters in damage detection. However, those demonstrated limitations in the classification of complex scenes, and of global feature representations on general. The latter cause problems when scene and image characteristics vary, which is typically the case between different disaster areas or in multi-temporal assessments. Therefore, the work described in Vetrivel et al. (2016b) moved towards descriptors that are more generalizable and invariant to image characteristics. The method was built on the Visual Bag of Words approach and focused on the detection of rubble, debris piles and severe spalling. The method performed well on individual UAV and also Pictometry data sets of Mirabello (Italy) and Port-au-Prince (Haiti), respectively, but also on a dataset that combined the two airborne datasets with transverse street-level images. The limitation of the method is that it is grid-based and can only identify general damage patches, i.e. grid cells affected by one or more of the damage types considered, while the detailed localisation and characterisation (size, shape, etc.) of damages of a specific type would be desirable.

\subsection{Deep learning}

Image classification used in damage mapping increasingly made use of machine learning, in particular SVM and Random Forest (Gong et al. 2016, Vetrivel et al. 2015a) or Adaboost (Gerke and Kerle 2011a). However, the features used were typically hand-crafted (such as HoG or Gabor), and emerging work had shown that in deep learning approaches convolutional neural networks $(\mathrm{CNN})$ could actually learn features and their representation directly from the image pixel values (Szegedy et al. 2015). Thus the damage detection work proceeded in this direction, hypothesizing that image classification would benefit from the micropropagation of $3 \mathrm{D}$ point cloud features. We applied a multiple-kernel-learning framework on several sets of diverse aerial images (Vetrivel et al. 2018), and showed that combining the radiometric and geometric information yields higher classification accuracies. The processing was based on Simple Linear Iterative Clustering (SLIC) superpixels, meaning that damage was again only identified in patches, though those were labelled with specific prediction scores. Of particular significance for the disaster response and search \& rescue focus of the projects was that the method demonstrated significant transferability. A model trained with a sufficient number of samples (e.g., trained before an actual event) performed well when then applied to a new disaster scene, supporting a rapid analysis without the need for extensive retraining.

Disaster scenarios are frequently characterised by imperfect image data availability, and a rapid response effort has to make do with what exists. In this respect it is valuable to be able to incorporate images of different types and scales into the training model. Duarte et al. (2018b) trained a CNN with different types of aerial imagery to classify post-disaster satellite data of Port-au-Prince. Although information coming from the different image resolutions evidently improved the model and classification accuracy, the approach still failed to capture smaller damage features. The work also focused on determining the effect of multi-scale information on the CNN activation layers as a proxy for improved damage recognition, while not allowing a detailed assessment of where the classification improvement originated in terms of false positives and negatives, or specific damage types. The work proceeded with a focus on multi-resolution feature fusion and its effect on building damage classification (Duarte et al. 2018a). It showed that such a fusion is useful and can improve the overall accuracy, though it also fails to show which specific damage types are identified, and how well they are captured.

Our contribution to RECONASS and INACHUS was centred on the use of highly detailed UAV images that provide views on all exterior parts of the surveyed buildings. Earlier work had shown how highly variable the expression of structural damage is in vertical and oblique data (Kerle and Hoffman 2013). The former essentially only considers the damage expressed in the roof, and in addition makes use of proxies such as debris piles for specific shadow configurations (for a recent review of those proxies see Ghaffarian et al. 2018). Significant additional information is also encoded in the façade information, as already explained in section 2.1. However, the OBIA-based approach tends towards overfitting and lacks the efficiency and transferability of deep learning. While a focus on façades is appealing, their actual delineation in imagery poses its own challenges, especially when considering aspects such as occlusion or environmental effects such as shadows (Figure 2).
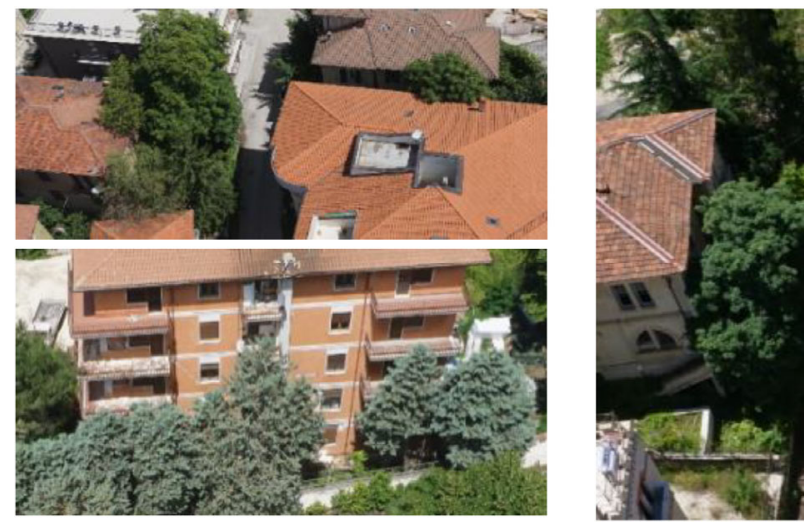

Figure 2. Problems for image processing posed by shadow and occlusion (Duarte et al. 2017).

Duarte et al. (2017) thus focused on developing an efficient method to extract façades that were subsequently assessed for damage using CNN. The approach made use of a point cloud calculated from vertical imagery acquired in an initial UAV survey. From the sparse point cloud the building form including its façades was hypothesised, which in turn was used to extract the actual façades from oblique UAV images. The patch-based damage classification had an overall accuracy of approximately $80 \%$, though the work also demonstrated the significant challenge of damage identification on façades, due to architectural complexities and associated diverse shadow patterns, but also occlusion (by external features such as vegetation, or internal ones such as balconies).

It stands to reason that some ambiguities can be resolved by analysing multi-perspective data (views of a given façade from different angles), but also by incorporating multi-temporal data where available. We tested those assumptions in a CNN framework and concluded that a multi-temporal approach with 3 views at each of the two epochs performed best (Duarte et al. in review), though also here smaller damage features eluded detection. 


\section{TESTS AND VALIDATON}

\subsection{Pilot experiments}

Both RECONASS and INACHUS included a number of pilot experiments, where first individual components or sets thereof, and later the entire systems were tested under relatively realistic conditions. For the already mentioned explosion experiments in Sweden we acquired data using a Aibot X6 Hexacopter carrying a Canon D600 camera with a Voigtländer $20 \mathrm{~mm}$ lens. In addition to reference data images were acquired after both the exterior and the interior blasts, with a ground sampling distance (GSD) of approximately $1.5 \mathrm{~cm}$. From those images detailed 3D point clouds were calculated and analysed. The data proved suitable to identity damage-related openings, such as infill walls damaged or blown-out by the blasts, as well as cracks and debris. Additionally, subtle façade deformations could be detected and quantified (Figure 3). It was also shown how a BIM model of the structure in 3D CityGML format could be automatically updated, both to visualise and catalogue detailed damage information. THW deployed a LEICA TM30 total station to survey the structure from 4 reference points, using 16 prisms mounted on the structure. While the total station has the advantage that a structure can be continuously monitored for minute deformations - critical when rescue personnel operates near or within weakened structured - the UAV-derived data provided damage data of comparable quality, with greater flexibility and lower cost, including the roof that ground-based surveys cannot see, and potentially operated from a safer distance. The building was further surveyed by a Riegl VZ400 terrestrial laser scanner, which also confirmed the high quality of the UAV-derived 3D models.

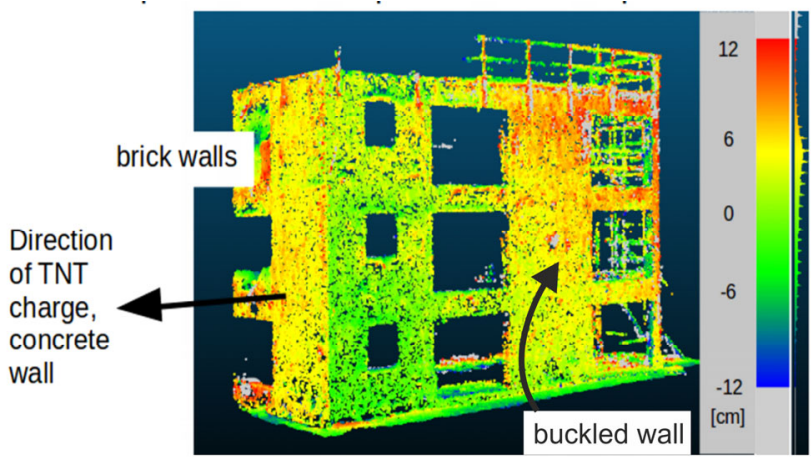

Figure 3. UAV-derived point clouds of reinforced concrete structure with brick in-fill walls subjected to exterior and interior detonations. Openings, cracks and debris piles were automatically detected, and also subtle deformation in the façades were detected.

For INACHUS pilot experiments were conducted at 4 different sites in France and Germany, and included buildings in the process of being demolished, as well as an urban search \& rescue training site (Training Base Weeze in Germany). In response to criticism by end users in RECONASS as to the high cost of the Aibot UAV (ca. 40,000 Euro), in INACHUS we used low-cost DJI drones (Phantom 4 and Mavic Pro). Following the research directions described in section 2.3, the work focused less on simple scene reconstruction, but on integration with other spatial data, as well as advanced data analysis, including with $\mathrm{CNN}$. For each of the pilots the building in question was also surveyed by ONERA using different UAV-borne laser scanners, as well as with a terrestrial laser scanner, to detect the respective strengths of the individual systems. The initial experiments with UAV-based laser scanners failed. First a Riegl VZ-1000 instrument (weight of about $10 \mathrm{~kg}$ ) was deployed on a Yamaha RMAX helicopter (weight $>60 \mathrm{~kg}$ ), though the acquired data suffered from artefacts and were not useful. Also data acquired with a Velodyne HDL32 (weight of only $1.3 \mathrm{~kg}$ ) deployed on a VARIO BENZIN helicopter (weight just under $10 \mathrm{~kg}$ ) proved unusable for damage detection, owing to the very unstable platform. For the final pilot a high quality Riegl VUX-1 was mounted on a stable DJI Matrice 600 hexacopter platform. The data were excellent, though the combined system is also very costly and requires expert knowledge for flight planning and executing, as well as data processing. We limited our mapping to the optical data acquired with the built-in cameras of the Phantom 4 and Mavic Pro (costs of $<2000$ Euro), and advanced along the computer vision and machine learning trajectory described above. The 3D data obtained from the optical imagery was of comparable quality to the VUX-1 data while also providing native colour information, better spatial detail, and full coverage also of façades (Figure 4). The expectation that the airborne laser data would patch the one principal weakness of photogrammetry, the inability to map dark interior spaces through openings, was also not met. The data on openings and connected interior spaces were primarily delivered from the tripod-mounted ground-based laser scanner, though here the limited flexibility and occlusion by the building's structural elements also prevented a complete mapping of openings.

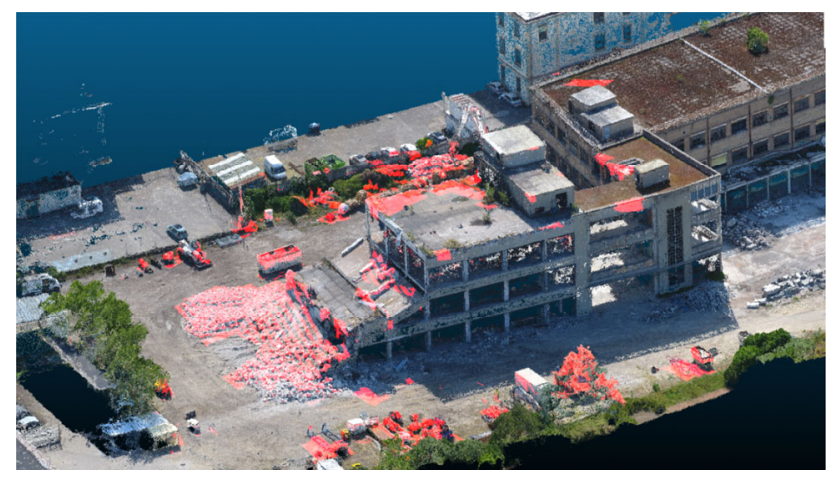

Figure 4. Point cloud representation of an INACHUS pilot structure in Lyon, France, calculated from optical imagery acquired with a low-cost commercial drone (Phantom 4, DJI), showing damage detected through machine learning (red).

While commercial UAV by DJI and other makers have clearly reached high levels of cost-benefit, stability and reliability, most are also not designed to be survey-grade instruments working in real time. For rapid search \& rescue support it is vital to provide usable information quickly. For that reason we also developed a procedure to process the data with minimal delay. The Mavic Pro allows images to be streamed during flight, which means that we built a procedure that (i) downloads images right after acquisition, (ii) builds a progressively extended 3D model of the scene using established structure from motion (SfM) methods, (iii) applies the machine learning algorithm to detect damage, and (iv) orthorectified the images using the $3 \mathrm{D}$ model. By the time the UAV lands after a maximum flight duration of about 25 minutes all processing is done and the damage map available. We built a smart phone app that allows this procedure to be executed together with a standard laptop (Figure 5). Details about the app and data processing workflow can be found in Nex et al., (2019). 

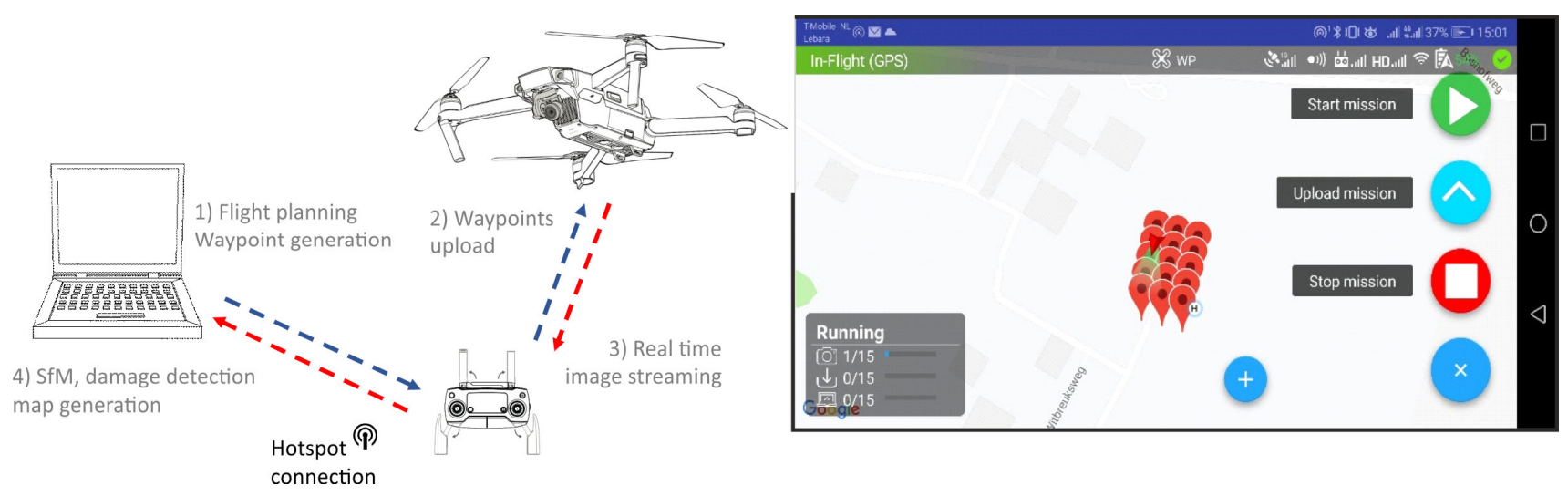

Figure 5. Work flow of the app developed for real-time damage mapping. Images are streamed to a laptop computer and processed immediately after acquisition. A CNN-based damage detection algorithm is applied, and a progressively built 3D model is used to orthorectify them. By the time the UAV lands an orthomosaic displaying the damage is finished.

\subsection{Validation}

At every pilot different end users were present and underwent a detailed assessment protocol of every tool produced and tested. At the final INACHUS pilot that took place in Roquebillière, France, in November 2018 a total of 25 end users from 8 countries participated, representing USAR teams and other civil protection organisations. They followed individual demonstrations of all technical tools developed, and graded them. Of all hard- and software or procedure solutions developed in INACHUS, the 3D mapping and damage detection with light-weight commercial UAV scored highest (overall 4.5 out of 5). The high score does not so much represent a high level of technical sophistication, but rather the simplicity, both in terms of off-the-shelf hardware and an automated flight planning and damage mapping routine. The end users especially liked the simple, low-cost approach that provided accurate and useful information in near-real time, without the need for a highly specialised operator.

\subsection{Limitations}

The end user assessment also revealed limitations of the developed damage mapping solution. Legal restrictions of drone deployment continue to pose challenges, though problems are less severe for lighter platforms, and in addition first responder and civil protection organisations tend to operate under different legal frameworks. A clear disadvantage of small multicopter UAV platforms is their comparatively small operating range and flight duration. The limited spatial scope of RECONASS and INACHUS matched their abilities well, but damage assessment over larger affected areas requires different solutions. Off-the-shelf UAV come equipped with high quality optical cameras, though the computer vision processing to generate 3D point clouds fails for dark image patches such as shadow or smaller building openings. For this reason openings and possible survival spaces in the pilot structures could not be mapped, and here active sensors have a clear advantage. Commercial UAV also tend to be closed and largely proprietary systems, meaning that it is not easily possible, if at all, to exchange or add sensors, or to install processing units such as a DJI Manifold or NVIDIA Jetson TX2 to push more autonomy in onboard image processing or dynamic flight path adjustment onto the drone.

\section{OUTLOOK AND NEW DEVELOPMENTS}

\subsection{Next steps in damage mapping}

Despite the progress that new platforms such as UAV and better image processing, in particular 3D reconstruction through advanced computer vision and deep learning, have generated, a range of challenges remains: (i) for all the sophistication of machine leaning approaches to recognise patterns and features, the specific effect of certain training labels remains unclear, challenging effort to optimise the training efficiency for specific damage features. Also, solutions developed to date still tend to be area/grid-based, highlighting damage in general, but not specific features. Work such as by Duarte et al. (2018b) also tends to focus on improving the overall classification accuracy, or on activation layers. From a user perspective more clarity in how a classifier perform in terms of reduced false positives or negatives is needed instead. (ii) traditional (vertical) damage was limited to information of the roof, supported by debris evidence and useful shadow signatures. The ability to map every surface plane of a building has generated a wealth of additional damage information. However, a disconnect remains between identifying a damage feature such as a crack or opening and its meaning and significance from a structural integrity or building function perspective. Damage indicators do not add up linearly to a per-building score, and the typical absence of information from the building's interior, but also an incomplete picture caused by occlusion, continue to pose a significant methodological challenge. (iii) in Duarte et al. (2017) we showed how data from an initial coarse vertical survey can be used to guide a more local assessment. Such a multi-scale approach needs to be better automated, with a UAV carrying out real-time coarse mapping until a damage candidate building is detected, which is then mapped in more detail. This needs to include a real-time assessment of the 3D reconstruction achieved, coupled with an autonomous decision as to whether acquire additional imagery, and from which position. (iv) in recent years there has been a surge in research on UAV-based indoor mapping, both with single platforms and swarms. Most make use of visual SLAM to map their GPS-denied environment (e.g., Bavle et al. 2018, Trujillo et al. 2018), or focusing on continuity mapping when transiting between outdoor and indoor places (Zhang et al. 2015). Others have experimented with localizing via sensors such as ultrasound (Paredes et al. 2018). (v) while damage assessment remains a relevant and timely research issues, in the age of aging 
infrastructure efforts have been spreading towards UAVsupported maintenance. This implies a number of challenges. Infrastructure is diverse and includes complicated indoor spaces such as chimneys (Quenzel et al. 2019), but also roads, tunnels and bridges. Solutions are emerging to carry out day-to-day monitoring to detect defects or signs of decay, but also damage after a disaster event or accident (e.g., Schweizer et al. 2018). Such works increasingly extends into another emerging line of development, (vi) blending UAV-based abilities with robotics and mechatronics solutions. Here UAV are not only used to map and mode infrastructure spaces, but also to carry actuator arms to place sensors for in-situ measurements (Jimenez-Cano et al. 2017, Sanchez-Cuevas et al. 2019), or to carry our limited repairs.

\subsection{Project continuation}

Since 2018 we have been working on H2020 project PANOPTIS (Development of a Decision Support System for increasing the Resilience of Transportation Infrastructure based on combined use of terrestrial and airborne sensors and advanced modelling tools; www.panoptis.eu). It focuses on developing a framework to make road infrastructure more resilient given present environmental hazards and climate change. Our work focuses on road surface and road corridor damage assessment to detect gradual signs of wear and decay, as well as the ability to respond rapidly to a disaster situation. We focus on the of emerging hybrid UAV platforms (here a DeltaQuad from vertical Solutions) that allows both corridor mapping of a fixed-wing platform and hovering for detailed mapping, and a Jetson TX2 will be used to advance data processing on the drone itself. $\mathrm{CNN}$-based methods are again applied for the damage feature detection.

In September 2019 we will start project INGENIOUS (The First Responder of the Future: a Next Generation Integrated Toolkit for Collaborative Response, increasing protection and augmenting operational capacity; www.ingenious-firstresponders.eu). Here we will focus on the use of drone swarms for indoor mapping to support first responders unknown and potentially dark, smoke-filled and hazardous indoor settings. The work will be done together with robotics partners and involve UAV platforms of different sizes and with different sensor load and ability, with focus on collaboration and optimization.

\section{CONCLUSIONS}

Structural damage mapping with remote sensing has been a continuous research problem for decades, and for rapid operational disaster response, such as through the Charter or Copernicus EMS, reliable automated methods continue to be lacking. The substantial progress that has been made in the last decade resulted primarily in rapid developments in UAV technology, computer vision, and in advanced image data processing with machine learning, in particular deep learning with CNN. This paper primarily focused on research achievements made in the context of two European-funded projects, RECONASS and INACHUS, though has also reviewed significant developments made by other groups during this time.

The paper has shown how image-derived 3D point clouds allow a highly detailed and accurate scene reconstruction, and how the coupling of the geometric information with the original image information allows very advanced feature recognition. Classifier training is also starting to overcome the challenge of in particular $\mathrm{CNN}$-based methods requiring millions of training samples. The development of unsupervised CNN approaches (such as Auto-encoders) or Generative Adversarial Networks (GAN) could represent a step forward in this direction. Newer approaches are improving the efficiency, but also the transferability of classifiers, critical to be able to respond quickly to a disaster event. Comprehensive tests with first responders and urban search \& rescue personnel showed that in particular solutions with light-weight off-the-shelf drones strike a very good compromise of high information quality and ready usability.

Developments continue at a rapid pace, with significant research efforts now being focused on UAV-based mapping in indoor settings, on UAVs also being equipped with mechatronic abilities to allow the deployment of additional sensors or to carry out repairs, though also newer networks allow more sophisticated and robust deep learning solutions. Nevertheless, more effort is needed to understand better the actual meaning and significance of specific damage evidence. In addition, UAV needed to become more autonomous to increase the efficiency of damage mapping operations. Finally, progress in the processing of UAV-based imagery, in particular through advanced machine learning, must eventually lead to fully automated and accurate damage mapping with optical satellite imagery.

\section{ACKNOWLEDGEMENTS}

This work was funded by the EU-FP7 projects RECONASS (grant no. 312718) and INACHUS (grant no. 607522), as well as H2020 project PANOPTIS (grant no. 769129). We thank Pictometry, Inc. for providing the Haiti and Italy imagery used in this study, and the DigitalGlobe Foundation (www.digitalglobefoundation.com) for providing satellite images on Italy and Ecuador.

\section{REFERENCES}

Bavle, H., Sanchez-Lopez, J.L., de la Puente, P., RodriguezRamos, A., Sampedro, C. and Campoy, P. (2018) Fast and robust flight altitude estimation of multirotor UAVs in dynamic unstructured environments using 3D point cloud sensors. Aerospace 5(3), 21.

Belabid, N., Zhao, F., Brocca, L., Huang, Y.B. and Tan, Y.M. (2019) Near-real-time flood forecasting based on satellite precipitation products. Remote Sensing 11(3), 18.

Corbane, C., Saito, K., Dell'Oro, L., Gill, S.P.D., Piard, B.E., Huyck, C.K., Kemper, T., Lemoine, G., Spence, R.J.S., Shankar, R., Senegas, O., Ghesquiere, F., Lallemant, D., Evans, G.B., Gartley Joaquin Toro, R.A., Ghosh, A., Svekla, W.D., Adams, B.J. and Eguchi, R.T. (2011) A comprehensive analysis of building damage in the 12 January 2010 Mw7 Haiti earthquake using high resolution satellite and aerial imagery. Photogrammetic Engineering and Remote Sensing 77(10), 9971009.

Cusicanqui, J., Kerle, N. and Nex, F. (2018) Usability of aerial video footage for 3-D scene reconstruction and structural damage assessment. Nat. Hazards Earth Syst. Sci. 18(6), 15831598 .

Dong, L.G. and Shan, J. (2013) A comprehensive review of earthquake-induced building damage detection with remote sensing techniques. ISPRS Journal of Photogrammetry and Remote Sensing 84, 85-99. 
Duarte, D., Nex, F., Kerle, N. and Vosselman, G. (2017) Towards a more efficient detection of earthquake induced façade damages using oblique UAV imagery. Int. Arch. Photogramm. Remote Sens. Spatial Inf. Sci. XLII-2/W6, 93100.

Duarte, D., Nex, F., Kerle, N. and Vosselman, G. (2018a) Multi-Resolution Feature Fusion for Image Classification of Building Damages with Convolutional Neural Networks. Remote Sensing 10(10), 1636.

Duarte, D., Nex, F., Kerle, N. and Vosselman, G. (2018b) Satellite image classification of building damages using airborne and satellite image samples in a deep learning approach. ISPRS Ann. Photogramm. Remote Sens. Spatial Inf. Sci. IV-2, 89-96.

Duarte, D., Nex, F., Kerle, N. and Vosselman, G. (in review) Multi-temporal image classification of earthquake induced façade damages with convolutional neural networks using aerial oblique imagery. Remote Sensing

Dubois, D. and Lepage, R. (2014) Fast and efficient evaluation of building damage from very high resolution optical satellite images. Ieee Journal of Selected Topics in Applied Earth Observations and Remote Sensing 7(10), 4167-4176.

Fernandez Galarreta, J., Kerle, N. and Gerke, M. (2015) UAVbased urban structural damage assessment using object-based image analysis and semantic reasoning. Natural Hazards and Earth System Sciences 15, 1087-1101.

Gerke, M. and Kerle, N. (2011a) Automatic structural seismic damage assessment with airborne oblique Pictometry (C) imagery. Photogrammetric Engineering and Remote Sensing 77(9), 885-898.

Gerke, M. and Kerle, N. (2011b) Graph matching in 3D space for structural seismic damage assessment. 2011 Ieee International Conference on Computer Vision Workshops (Iccv Workshops).

Ghaffarian, S., Kerle, N. and Filatova, T. (2018) Remote sensing-based proxies for urban disaster risk management and resilience: A review. Remote Sensing 10(11), 30.

Gong, L.X., Wang, C., Wu, F., Zhang, J.F., Zhang, H. and Li, Q. (2016) Earthquake-induced building damage detection with post-event sub-meter VHR TerraSAR-X Staring Spotlight imagery. Remote Sensing 8(11), 21.

Grenzdorffer, G.J., Guretzki, M. and Friedlander, I. (2008) Photogrammetric image acquisition and image analysis of oblique imagery. Photogrammetric Record 23(124), 372-386.

Grünthal, G. (ed) (1998) European Macroseismic Scale 1998 (EMS-98), Cahiers du Centre Européen de Géodynamique et de Séismologie, Centre Européen de Géodynamique et de Séismologie.

Jimenez-Cano, A.E., Heredia, G. and Ollero, A. (2017) Aerial manipulator with a compliant arm for bridge inspection, pp. 1217-1222.

Kerle, N. (2015) Disasters : risk assessment, management, and post - disaster studies using remote sensing. In: Remote sensing of water resources, disasters, and urban studies / edited by P.S. Thenkabail. Boca Raton: CRC Press, 2015. ISBN: 978-1-48221792-6 (Remote sensing handbook, 3) pp. 455-481.

Kerle, N. and Hoffman, R.R. (2013) Collaborative damage mapping for emergency response: the role of Cognitive Systems
Engineering. Natural Hazards and Earth System Sciences 13(1), 97-113.

Li, L.L., Liu, X.G., Chen, Q.H. and Yang, S. (2018) Building damage assessment from PolSAR data using texture parameters of statistical model. Computers \& Geosciences 113, 115-126.

Lu, C.H., Ni, C.F., Chang, C.P., Yen, J.Y. and Chuang, R.Y. (2018) Coherence difference analysis of Sentinel-1 SAR interferogram to identify earthquake-Iinduced disasters in urban areas. Remote Sensing 10(8), 21.

Mitomi, H., Yamzaki, F. and Matsuoka, M. (2000) Automated detection of building damage due to recent earthquakes using aerial television images, pp. 401-406, GIS Development, Taipei, Taiwan.

Nex, F., Duarte, D., Steenbeek, A. and Kerle, N. (2019) Towards real-time building damage mapping with low-cost UAV solutions. Remote Sensing 11(3), 287.

Novikov, G., Trekin, A., Potapov, G., Ignatiev, V. and Burnaev, E. (2018) Satellite imagery analysis for operational damage assessment in emergency situations, pp. 347-358, Springer International Publishing, Cham.

O’Rourke, T.D., Bonneau, A.L., Pease, J.W., Shi, P. and Wang, Y. (2006) Liquefaction and ground failures in San Francisco. Earthquake Spectra 22(S2), 91-112.

Paredes, J.A., Alvarez, F.J., Aguilera, T. and Villadangos, J.M. (2018) 3D indoor positioning of UAVs with spread spectrum ultrasound and time-of-flight cameras. Sensors 18(1), 15.

Quenzel, J., Nieuwenhuisen, M., Droeschel, D., Beul, M., Houben, S. and Behnke, S. (2019) Autonomous MAV-based indoor chimney inspection with 3D laser localization and textured surface reconstruction. Journal of Intelligent \& Robotic Systems 93(1-2), 317-335.

Sanchez-Cuevas, P.J., Ramon-Soria, P., Arrue, B., Ollero, A. and Heredia, G. (2019) Robotic system for inspection by contact of bridge beams using UAVs. Sensors 19(2), 17.

Schweizer, E.A., Stow, D.A. and Coulter, L.L. (2018) Automating near real-time, post-hazard detection of crack damage to critical infrastructure. Photogrammetric Engineering and Remote Sensing 84(2), 76-87.

Sublime, J. and Kalinicheva, E. (2019) Automatic post-disaster damage mapping using deep-learning techniques for change detection: Case study of the Tohoku Tsunami. Remote Sensing 11(9), 20.

Szegedy, C., Liu, W., Jia, Y.Q., Sermanet, P., Reed, S., Anguelov, D., Erhan, D., Vanhoucke, V., Rabinovich, A. and Ieee (2015) 2015 IEEE Conference on Computer Vision and Pattern Recognition, pp. 1-9, IEEE, New York.

Trujillo, J.C., Munguia, R., Guerra, E. and Grau, A. (2018) Visual-based SLAM configurations for cooperative multi-UAV systems with a lead agent: An observability-based approach. Sensors 18(12), 30.

Vetrivel, A., Duarte, D., Nex, F., Gerke, M., Kerle, N. and Vosselman, G. (2016a) Xxiii ISPRS Congress, Commission Iii. Halounova, L., Schindler, K., Limpouch, A., Pajdla, T., Safar, V., Mayer, H., Elberink, S.O., Mallet, C., Rottensteiner, F., Bredif, M., Skaloud, J. and Stilla, U. (eds), pp. 355-362.

Vetrivel, A., Gerke, M., Kerle, N., Nex, F. and Vosselman, G. (2018) Disaster damage detection through synergistic use of 
deep learning and 3D point cloud features derived from very high resolution oblique aerial images, and multiple-kernellearning. ISPRS Journal of Photogrammetry and Remote Sensing 140, 45-59.

Vetrivel, A., Gerke, M., Kerle, N. and Vosselman, G. (2015a) Identification of damage in buildings based on gaps in 3D point clouds from very high resolution oblique airborne images. ISPRS Journal of Photogrammetry and Remote Sensing 105, 61-78.

Vetrivel, A., Gerke, M., Kerle, N. and Vosselman, G. (2015b) Pia15+Hrigi15 - Joint ISPRS Conference, Vol. I. Stilla, U. and Heipke, C. (eds), pp. 261-268.

Vetrivel, A., Gerke, M., Kerle, N. and Vosselman, G. (2016b) Identification of Structurally Damaged Areas in Airborne Oblique Images Using a Visual-Bag-of-Words Approach. Remote Sensing 8(3).

Yamazaki, F., Yano, Y. and Matsuoka, M. (2005) Visual damage interpretation of buildings in Bam City using quickBird images following the 2003 Bam, Iran, earthquake. Earthquake Spectra 21, S328-S336.

Zhang, X., Xian, B., Zhao, B. and Zhang, Y. (2015) Autonomous flight control of a nano quadrotor helicopter in a GPS-denied environment using on-board vision. IEEE Transactions on Industrial Electronics 62(10), 6392-6403. 